\title{
Matching Composite Sketches to Facial Photos using Component-based Approach
}

\author{
Archana Uphade \\ P.G.Student \\ Department of Computer Engineering \\ Late G.N.Sapkal College of Engineering, \\ Anjaneri, Nasik
}

\author{
J.V. Shinde \\ Assistant Professor \\ Department of Computer Engineering \\ Late G.N.Sapkal College of Engineering, \\ Anjaneri, Nasik
}

\begin{abstract}
Face recognition has an important application in criminal investigation. Previous research on sketch recognition focused on matching sketches drawn by professional artists. There has been number of representation methods are used to solve the problem of matching facial sketches to photographs.

In the proposed system composite sketches are synthesized using one of the several facial composite software systems. A component-based representation (CBR) approach is used to measure the similarity between a composite sketch and mugshot photograph. First detect the facial landmarks in composite sketches and face photos using an active shape model (ASM) followed by computing length between elements. Features are then extracted for each facial component using multiscale local binary patterns (MLBPs), and per component similarity are calculated. In proposed system per component features are measured and compared with the features of the mugshot gallery set for matching. Depending on the component matching images will be display in sorted order.
\end{abstract}

\section{General Terms}

Recognition, Algorithm, Detection, Performance, Approach.

\section{Keywords}

Component-based, Composite-sketch, Forensic sketch, Heterogeneous, Modality gap.

\section{INTRODUCTION}

Recently face recognition is one of the most studied areas in computer vision. Also it has tremendous applications in criminal investigation. As a use of such application increases emerging technologies also improved to matching sketches to facial photographs.

In many cases photograph of the suspect is not available, in these cases police assist an artist to draw the sketches as per the description provided by an eye-witness. But artist required a large amount of training in case drawing the sketch therefore it becomes time consuming process. So due to budgetary reason many law-enforcement agencies now using composite software to synthesize the sketch.

There are several methods for matching hand drawn sketches to photographs are used in the literature, but there has been only limited research on matching computer generated composites to mugshot photographs. This work aims to develop a system that can successfully match composite sketches to mugshots.

\section{LITERATURE SURVEY}

\subsection{Viewed Sketch Face Recognition}

Due to improving accuracy Viewed sketch recognition has most importance.

Viewed sketch-based face recognition is based on the synthesis, projection and feature-based methods.

\subsection{Synthesis-based approaches}

This synthesis based approach focuses on method that takes a photo from corresponding sketch or vice-versa. An eigensketch transformation algorithm is used to convert a photo into sketch by [Tang and Wang 2002].Classification can be used on obtained eigenskecth feature. The discrepancies between photo and sketch are reduced by the eigensketch transformation algorithm.

Based on the image patches photos are converted into sketches using A Local Linear Embedding (LLE) method [Liu et al.2005]. Those sketches are geometry preserving synthetic sketches. For each image patch to be converted, it finds its nearest neighbours in the training set, and uses their corresponding sketch patches to synthesize the sketch patch. By developing an advance approach to synthesize local face image at different scales using a different technique Tang and Wang [Wang and Tang 2009] further improved [Liu et al. 2005] like a Markov Random Fields (MRF). In later, instead of independently which used in [Liu et al. 2005] a multi-scale MRF learns local patches and scales jointly.

\subsection{Projection based approaches}

In projection based approach a main strategy is to find a lower-dimensional sub-space so that two modalities can be directly compare.

In later Lin and Tang [Lin and Tang 2006] proposed method of a linear transformation which can be used between different modalities (sketch/photo), called common discriminate feature extraction (CDFE). Using this method, images from two modalities are projected into a common feature space so that matching can be efficiently performed.

\subsection{Feature based approaches}

This approach focuses on designing a feature descriptor for each image that is invariant to the modality, while being variant to the identity of the person rather than matching photos into sketches, or both into a common subspace. Scale invariant feature transform (SIFT), Gabor transform, Histogram of Averaged Oriented Gradients (HAOG) and Local Binary Pattern (LBP) this are most widely used image feature descriptor. Once sketch and photo images are encoded using these descriptors, they may be matched directly. 
Depending on the invariant SIFT-features [Lowe 2004].Klare et al. [Klare and Jain 2010b] proposed the first direct sketch/photo matching method. Based on the magnitude, orientation and spatial distribution of the image gradients, SIFT features provide a compact vector representation of an image patch [Klare and Jain 2010b]. SIFT feature vectors are first sampled uniformly from the face images and mixed together separately for sketch and photo images. Euclidean distance computed between concatenated SIFT feature vectors of sketch and photo images for NN matching.

\subsection{Composite sketch based face recognition}

These studies focused on face recognition using composite sketches which are synthesized by composite software system.

To represent sketches Yuen et al. [Yuen and Man 2007] uses the both features i.e. local and global. In the recognition phase this face recognition method required user input in the form of relevance feedback. The second two focus on holistic [Klare and Jain 2013] representation.

Holistic method [Klare and Jain 2013] uses similarities between local features Computed on uniform patches across the entire face images. Later translate it into a facial sketch/mug shot into 154 uniform patches, SIFT [Lowe 2004] and multi-scale local pattern (MLBP) [Ojala et al. 2002] invariant features are extracted from each patch. With this feature encoding, as improved version of the common representation from [Klare and Jain 2010b] is applied, later RS-LDA [Wang and Tang 2006b] is applied to generate a discriminative subspace for NN matching with cosine distance. The score generated by each feature and patch are used for final recognition.

\section{PROPOSED SYSTEM}

A component based representation (CBR) approach is used for matching composite sketches to facial photographs.

The proposed system consists of the following steps:

\section{A. Face Normalization}

The law enforcement agencies maintain the photograph of the suspect, but that photos may not be in frontal pose. There may be some variation in face pose. To handle these variations of photo a rotation and scaling is used to transfer a photo into frontal pose, called as normalization. Same steps are applied to composite sketches.

\section{B. Facial Component Localization}

After normalization facial elements localization is done using a Viola-Jones algorithm and active shape model (ASM) followed by computing length between elements. In the proposed system a face is divided into different facial components like eye, mouth and nose. Component is localized with a rectangle.

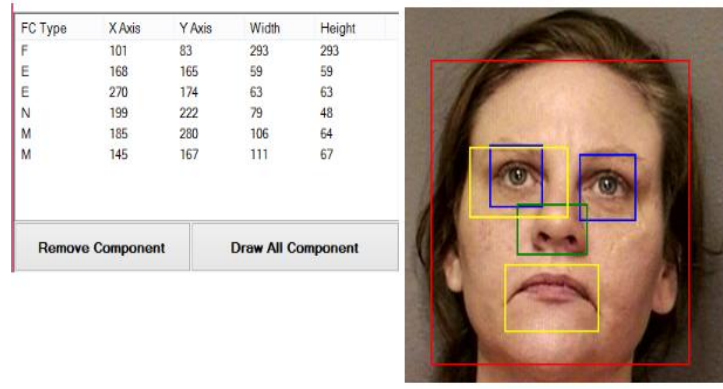

Fig 1: Component localization using Viola-Jones algorithm

In above figure facial components are separated with region values. Component information of mugshots are calculated and stored in the database for matching with the composite sketches.

\section{Component Feature Extraction}

Crucial landmarks are first determined and then Multiscale LBP algorithm is used for extracting window based features for eyes, nose, mouth etc. Once the features have been extracted, the dimension of the features can be reduced using feature reduction algorithms such as Principal Component Analysis. The features thus reduced are stored in database. Same process is also applied to the test image (sketch) and given to the classifier (Neural Network - Supervised) which is trained by the training set from the database features.

\section{Component Similarity and Fusion}

In proposed face retrieval system a component-based approach is used for face recognition. In this set of facial components are maintained for matching. For comparing whole input image with the image from gallery set, first compare each facial component from the data set of facial components. User can provide input image in the form of sketch or facial sketch component. When any new image comes for matching then extract the features of it and then compare the each facial component with data set of facial component. For each facial component will get matching image from gallery set. Depending on the facial component sorted images from dataset will be display as a output.

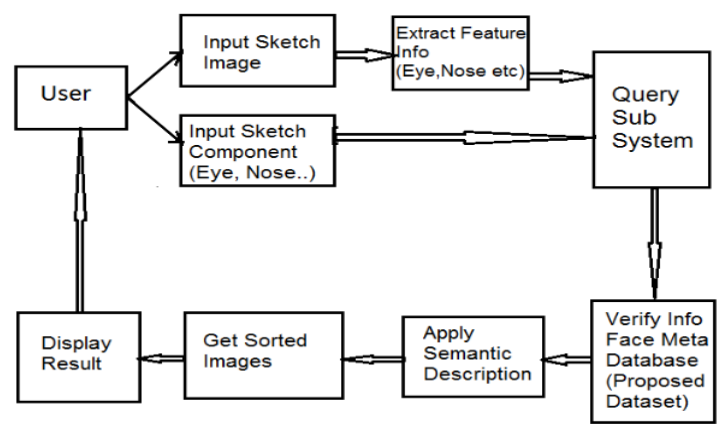

Fig 2: Block diagram of proposed system

Fig 2 shows that user can provide input image in the form of sketch image or sketch component to the recognition system. Recognition system extracts the feature information such as facial components like eye, nose etc. Query sub system verify the face meta database against the mugshot gallery set. In dataset feature 
information is already stored for each facial component at the time of training system. Then by comparing features sorted ordered matching images will get for given input as output result.

\section{ALGORITHMIC STRATEGY 4.1 CBR Technique for Pre-processing}

A. Pre-processing of mugshot using a geometric transformation, image enhancement and colour space conversion.

B. Facial elements localization using a Viola-Jones algorithm and active shape model (ASM) followed by computing length between elements.

C. Per component feature extraction using nearest neighbour symmetry and matrix normalize crosscorrelation.

D. Per component similarity measurement, followed by score normalization and fusion.

\subsection{Component localization Steps}

1. Detect the face.

2. Detect the components modes in the face region.

3. $\operatorname{if}(\mathrm{c}(\mathrm{x})>0)$ then loop components $\mathrm{c}(\mathrm{x})$, extract the features related to component by their pixel.

4. Constrain each component at the mode with maximal weight; search an initial shape S0 using Eqn.

for $\mathrm{k}=1$ to $\mathrm{n}$

(i) For each component, perform discriminative search.

Determine the searching direction by direction classifiers.

Move the component to an appropriate position along the direction.

(ii) Search with new component constraints; get the shape Sk by Eqn.

(iii) Evaluate the shape score $\mathrm{f}(\mathrm{Sk})$.

The output shape $S k=\operatorname{maxSk} f(S k)$.

else

Reject the component and goto the next image

\subsection{Extraction Steps using MLBP Algorithm}

1. Feature extraction - Crucial landmarks are first determined and then Multiscale LBP algorithm is used for extracting window based features for eyes, nose, mouth etc.

2. Dimension Reduction - After the features have been extracted, the dimension of the features can be reduced using feature reduction algorithms such as Principal Component Analysis.

3. Data base maintenance - The features thus reduced are stored in database.

4. Classifier - The test image (sketch) is also followed through the steps from 1-3 and given to the classifier (Neural Network - Supervised) which is trained by the training set from the database features.
5. Result - The higher matching face is then displayed. Another four such faces will also be displayed which have higher matching percentage below the first face according to the matching percentage.

Identification of composite sketch takes place for $(\mathrm{N})$ number of face images.

\subsection{Similarity Measurement}

Steps to match composite sketch to mug shot images are as follow:

1. Facial features for each face image/composite sketch are extracted $(F)$, then their length or width or area ratio is calculated and these extracted facial features are represented as a feature vector $(\mathrm{V})$. If there are $\mathrm{n}$ images then there will be $n$ number of vectors.

2. Mean (M) for every vector is computed and subtracted from each feature vector for centering of the feature vectors $(\mathrm{F}=\mathrm{M}-\mathrm{V})$.

3. K-NN classifier is used to identify facial composite sketch from existing database with $\mathrm{K}=5$ and Euclidean Distance is used.

4. Finally we get best matching five face images with composite sketch.

\section{RESULT AND ANALYSIS}

When input sketch image is provided to the system, it provides the matching photo images to given sketch. Recognition rate is different for different facial component. Matching performance of different component for rank-1 to rank-100 is calculated and shown in below graph.

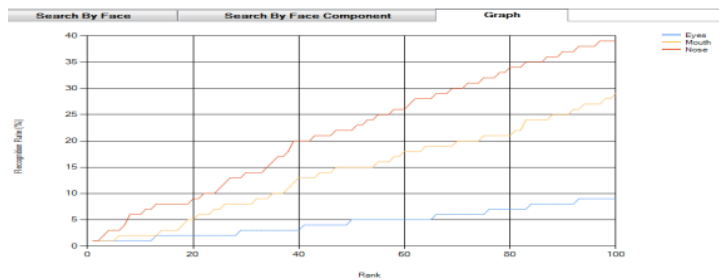

Fig 3: Performance of proposed approach for different ranks.

The pattern/structure of the eyes is more complicated than the other components. This makes it more difficult to find exact matching among the eye component from the dataset. Therefore, in above graph eye component gives the lower recognition rate for sketch to face photo matching.

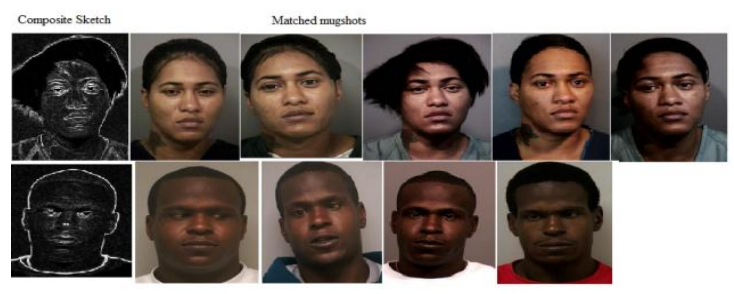

Fig 4: Examples of rank-1 retrievals for matching composite-photo pairs with the proposed approach utilizing gender information that correctly matched composites-photo pairs. 
In this proposed system, matching is get for composite sketch to facial photos efficiently. Following example shows matching pairs of composite face-photo.

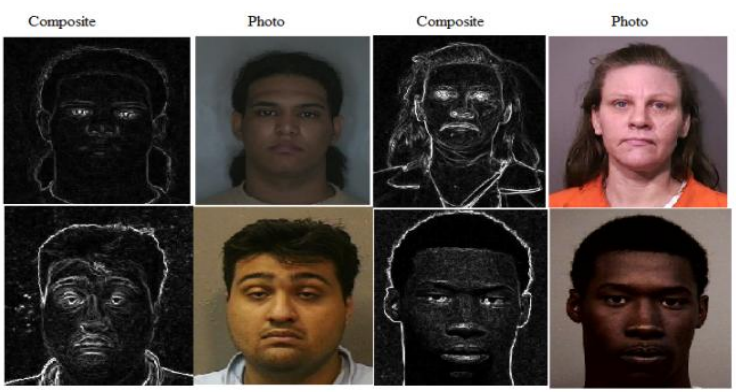

Fig 5: Examples of matching composite face-photo pairs with the proposed approach for MEDS-I dataset.

Also, proposed approach gives better result for one facial composite component. Following example shows for eyes, nose etc composite components photo matching is possible.

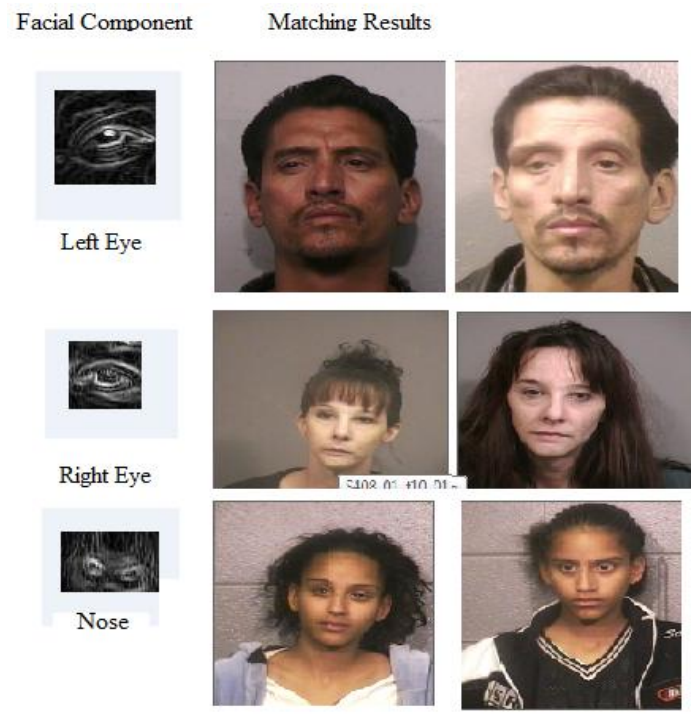

Fig 6: Examples for matching facial composite component with photo pairs using the proposed approach.

Precision: The precision is defined as the ratio of the number of relevant images retrieved and the total number of images in the dataset.

Sometimes for more than one person any facial component might be same. In this case result gives the different images for one person with same facial component. In this case precision rate is low.

Recall: Recall rate is defined as the ratio of number of relevant images retrieved and to the total number of relevant images in the dataset.

Matching is performed based on the similarity of the facial components. Recall is depends on the higher percentage matching of the components. Fig.4. shows comparison result for existing and proposed system with precision and recall values.

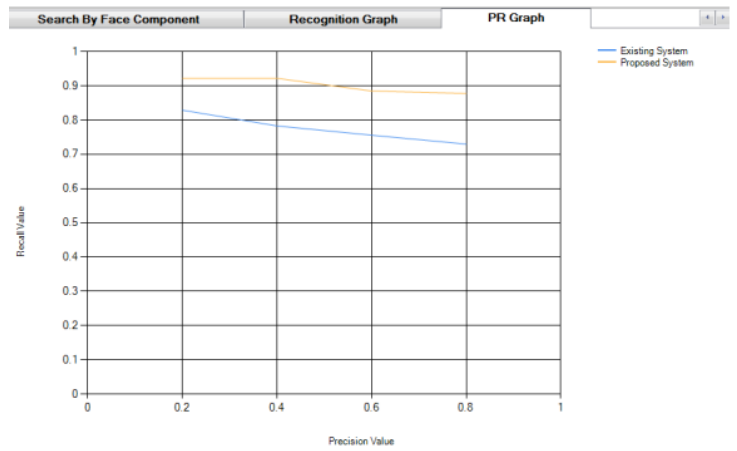

Fig 7: Comparison Result for Existing (Hu Han, Brendan F. Klare, Kathryn Bonnen, and Anil K. Jain) and Proposed System Representing Precision Recall values for MEDS-I Dataset.

\section{CONCLUSION AND FUTURE SCOPE}

The main goal of this work is to provide matching to possible suspect with mug shot gallery set.

Thus this system solves the challenging heterogeneous problem of matching composite sketches to mug shot gallery. Component based representation method address the modality gap between composite sketch and photo image efficiently. A Component based approach gives better performance due to the accuracy of synthesized sketches. Features are then extracted for each facial component using multiscale local binary patterns (MLBPs), and per component similarity are calculated. Proposed system finds the matching result based on the one facial component in that per component features are measured and compared with the features of the mugshot gallery set. Depending on the component matching images will be display in sorted order.

In future work, we would like to study the aging problem in composite-to-photo matching using the proposed approach.

\section{REFERENCES}

[1] Hu Han, Brendan F. Klare, Kathryn Bonnen, and Anil K. Jain. "Matching Composite Sketches to Face Photos: A Component-Based Approach" IEEE Transactions on Information Forensics And SECURITY, VOL. 8, NO. 1, JANUARY 2013.

[2] Brendan F. Klare and Anil K. Jain.2013."Heterogeneous Face Recognition Using Kernel Prototype Similarities". TPAMI (2013), 1410-1422.

[3] H. Han, S. Shan, X. Chen, S. Lao, and W. Gao, "Separability oriented preprocessing for illumination-invariant face recognition," in Proc. Eur. Conf. Computer Vision, 2012, pp. 307-320.

[4] B. Klare, Z. Li, and A. Jain, "Matching forensic sketches to mug shot photos," IEEE Trans. Pattern Anal. Mach. Intell., vol. 33, no. 3, pp. 639-646, Mar. 2011.

[5] Handbook of Face Recognition, S. Z. Li andA.K. Jain, Eds., 2nd ed. New York: Springer, 2011.

[6] W. Zhang, X. Wang, and X. Tang, "Coupled information-theoretic encoding for face photo- 
sketch recognition," in Proc. Conf. Computer Vision and Pattern Recognition, 2011, pp. 513-520.

[7] A. Sharma and D. Jacobs, "Bypassing synthesis: PLS for face recognition with pose, low-resolution and sketch," in Proc. IEEE Conf. Computer Vision and Pattern Recognition, 2011, pp. 593-600.

[8] H. Han, S. Shan, L. Qing, X. Chen, and W. Gao, "Lighting aware preprocessing for face recognition across varying illumination," in Proc. Eur. Conf. Computer Vision, 2010, pp. 308-321.

[9] X. Wang and X. Tang, "Face photo-sketch synthesis and recognition," IEEE Trans. Pattern Anal. Mach. Intell., vol. 31, no. 11, pp. 1955-1967, Nov. 2009.

[10] X. Wang and X. Tang, "Face photo-sketch synthesis and recognition," IEEE Trans. Pattern Anal. Mach. Intell., vol. 31, no. 11, pp. 1955-1967, Nov. 2009.

[11] Z. Lei and S. Li, "Coupled spectral regression for matching hegerogeneous faces," in Proc. IEEE Conf. Computer Vision and Pattern Recognition,2009, pp. 1123-1128.

[12] H. Han, S. Shan, X. Chen, and W. Gao, "Illumination transfer using homomorphic wavelet filtering and its application to light-insensitive face recognition," in Proc. Automatic Face and Gesture Recognition, 2008, pp. 1-6.

[13] X. Gao, J. Zhong, J. Li, and C. Tian, "Face sketch synthesis algorithm based on E-HMM and selective ensemble," IEEE Trans. Circuits Syst. Video Technol., vol. 18, no. 4, pp. 487-496, Apr. 2008.

[14] P. C. Yuen and C. H. Man. 2007."Human Face Image Searching System Using Sketches".IEEE Transaction on Systems, Man and Cybernetics, Part A: System and Humans (TSMC) (2007), 493-504.

[15] P. C. Yuen and C. H. Man, "Human face image searching system using sketches," IEEE Trans. Syst., Man, Cybern. A, Syst. Humans, vol. 37, no. 4, pp. 493-504, Jul. 2007.
[16] P. C. Yuen and C. H. Man, "Human face image searching system using sketches," IEEE Trans. Syst., Man, Cybern. A, Syst. Humans, vol. 37, no. 4, pp. 493-504, Jul. 2007.

[17] G.Wells and L. Hasel, "Facial composite production by eyewitnesses," Current Directions Psychol. Sci., vol. 16, no. 1, pp. 6-10, Feb. 2007.

[18] D. Lin and X. Tang, "Inter-modality face recognition," in Proc. Eur. Conf. Computer Vision, 2006, pp. 13-26.

[19] D. Lin and X. Tang, "Recognize high resolution faces: From macrocosm to microcosm," in Proc. IEEE Computer Vision and Pattern Recognition, 2006, pp. 1355-1362.

[20] D. Lin and X. Tang, "Inter-modality face recognition," in Proc. Eur. Conf. Computer Vision, 2006, pp. 13-26.

[21] D. Lin and X. Tang, "Recognize high resolution faces: From macrocosm to microcosm," in Proc. IEEE Computer Vision and Pattern Recognition, 2006, pp. 1355-1362.

[22] P. Sinha, B. Balas, Y. Ostrovsky, and R. Russell, "Face recognition by humans: Nineteen results all computer vision researchers shouldknow about," Proc. IEEE, vol. 94, no. 11, pp. 1948-1962, Nov. 2006.

[23] D. Mcquiston, L. Topp, and R. Malpass, "Use of facial composite systems in US law enforcement agencies," Psychology, Crime and Law, vol. 12, no. 5, pp. 505-517, 2006.

[24] C. Frowd, D. Carson, H. Ness, D. McQuiston, J. Richardson, H. Baldwin, and P. Hancock, "Contemporary composite techniques: The impact of a forensically-relevant target delay," Legal Criminol. Psychol., vol. 10, no. 1, pp. 63-81, Feb. 2005. 